\title{
Análisis de las bases filosóficas de las aportaciones de Pierre Bourdieu a la Teoría de la Educación
}

\section{Analysis of the philosophical foundations of Pierre Bourdieu's contributions to the Educational Theory}

\author{
Marcos SANTOS GÓMEZ \\ Universidad de Granada
}

Recibido: Mayo 2012

Aceptado: Octubre 2012

\section{Resumen}

El objetivo de este trabajo es analizar filosóficamente la crítica de Pierre Bourdieu a la concepción idealista de la razón y del sujeto para aplicarla al campo de la Teoría de la Educación. Partimos de una amplia revisión bibliográfica. Las conclusiones inciden en la imposibilidad de comprender la educación escindida de su componente material-social y apuntan a la necesidad de incluir siempre operativamente lo social y lo histórico como dimensiones constituyentes de la persona. Frente al idealismo del sujeto moderno cartesiano, es preciso que tanto el educador como el investigador sean conscientes de la materia social que los constituye.

Palabras clave: Teoría de la Educación, Filosofía de la Educación, Sociología de la Educación, Pierre Bourdieu, Escuela, Modernidad.

\begin{abstract}
The aim of this paper is analyzing philosophically the criticism of Pierre Bourdieu to the idealistic conceptions of reason and human being. This critique is applied to some topics of Theory of Education. So we have done a comprehensive review of specialized literature. The findings point to the impossibility of understanding education as separated from its socialmaterial component, and thus it is always necessary to include operationally the constitutive social and historical dimension of person. Besides, in opposition to the idealism of the modern Cartesian subject, it is necessary that both, the educator and the researcher, be conscious of their own social component.
\end{abstract}

Keywords: Educational Theory, Educational Philosophy, Educational Sociology, Pierre Bourdieu, School, Modernity. 


\section{Elementos de una teoría de la educación materialista}

Pierre Bourdieu, sociólogo francés de formación filosófica, abordó en varias obras famosas el campo de la educación (v. g. Bourdieu y Passeron, 2001). Se le considera, de hecho, uno de los principales sociólogos de la educación del siglo XX, de gran influencia en el análisis de los sistemas escolares y en el desarrollo de las teorías que a partir de él se han denominado "reproductivistas" en la pedagogía. En este trabajo, vamos a mostrar que su sociología puede ayudar a la Teoría de la Educación a tener en cuenta, como resulta imprescindible, el suelo de lo social donde se realiza en gran medida lo humano y que constituye a la persona.

Si acudimos a los clásicos del pensamiento pedagógico, Bourdieu atina mejor en cuanto a la asunción de la materialidad de lo humano y de lo educativo que por ejemplo otro clásico del pensamiento educativo como es John Dewey, como se explica en el trabajo de M. Santos (2011b). Creemos de hecho que ceñirse consecuentemente a esta materialidad puede ayudar a solventar algunas de las deficiencias que demuestran determinados enfoques pedagógicos a la hora de considerar la educación en valores y que son señaladas por García del Dujo y Mínguez (2011).

Hay que enfatizar que a la focalización de la pedagogía en lo corporal, que responde también a una cierta corrección de tipo materialista a la hora de estudiar la educación, se ha dedicado en los últimos años un importante esfuerzo investigador en el mundo de la Teoría de la Educación en España (Prieto, 2011). En una línea crítica también con el excesivo racionalismo con el que se aborda a veces el Yo y el sujeto en ciertas teorías pedagógicas, deseamos destacar el enfoque del profesor López Herrerías, que apunta a la preeminencia de lo ético-afectivo sobre lo estrechamente cognitivo (López Herrerías, 2010). Además, el discurso de una filosofía de la educación liberadora que se desarrolla en algunos trabajos recientes tiene como punto de partida precisamente la asunción de la materialidad (histórica y social) de lo humano (Santos, 2010, 2011a).

Bourdieu entronca con una tradición epistemológica que en el pensamiento francés se ha preguntado por las condiciones históricas de la racionalidad científica. Esta tradición nos remite a Comte y Durkheim y podemos quizás hacerla extensiva hasta Foucault. Dentro de esta línea de autores próximos o pertenecientes a la sociología cabe situar sus principales influencias, dentro y fuera de Francia: Marx, Weber y Durkheim (Castón, 1996: 77-78). Esta veta en el pensamiento francés se contrapone a otra que apunta a "las condiciones ontológicas que hacen posible el acontecer de la significación" (Moreno y Vázquez, 2006: 9). Aquí ubicaríamos por ejemplo a Sartre, Marcel, Merleau-Ponty ${ }^{1}$ hasta Ricoeur. La primera línea se caracteriza por asumir un análisis de tipo científico del objeto, frente al afán de radicalidad que aproxima a los autores de la segunda línea a lo artístico y literario. En éstos se da una apuesta

\footnotetext{
${ }^{1}$ A pesar de sus discrepancias con la fenomenología, en lo que se refiere a la corporalidad, al cuerpo que "piensa" y a la inserción práctica del cuerpo en su medio social, existen notorias semejanzas entre Bourdieu y Merleau-Ponty que han sido señaladas (Ferrante, 2008a). Otro trabajo, que también indica sus divergencias y similitudes con vistas a elaborar una síntesis de ambos es el de Marcoulatos (2001).
} 
metodológica por la fenomenología que persigue mostrar las estructuras fundamentales de lo óntico, es decir, ir de lo óntico a lo ontológico, por lo que la historicidad es algo que aunque se asume en los entes, se intenta trascender (Sartre, Heidegger). Buscan, por tanto, un trascender que no se daría en los autores más próximos al pensamiento sociológico. En este sentido, Bourdieu se halla en una opción epistemológica que lo aproxima a la corriente analítica en la filosofía, pero que se separa al mismo tiempo de corrientes fuertemente objetivistas que niegan la mediación de un sujeto entre la estructura social y las acciones, como es el estructuralismo de Levi-Strauss (Ferrante, 2008b).

Además, la concepción pragmatista de los usos del lenguaje a la que apunta el sociólogo francés en sus investigaciones recuerda a la tradición del segundo Wittgenstein o Austin. Serán las reglas de juego asumidas institucionalmente las que van creando una realidad que el sociólogo intenta describir. José Medina (2003), por ejemplo, interpreta la concepción epistemológica del segundo Wittgenstein como contextualismo social, argumentando que es estrechamente similar a la noción de doxa epistémica de Bourdieu, a la que nos referiremos más adelante.

Bourdieu emprende una crítica histórica de la razón que supone una solución a la dicotomía contemporánea de la opción obligada entre razón o historia. Se trata de dilucidar las reglas de juego del campo donde se da el pensamiento y la pesquisa científica. "Sólo advirtiendo los determinismos sociales que sesgan la mirada del filósofo o del investigador social es posible avanzar en la conquista de la autonomía y por lo tanto de la universalidad en el campo considerado" (Moreno y Vázquez, 2006: 16). Todo esto sería un procedimiento de criba que garantizaría no tanto la disolución de la crítica, sino precisamente la posibilidad de un campo autónomo donde pueda ejercerse. Así pues, en la polémica en torno a la Ilustración, Bourdieu se decanta no tanto por combatirla y arrojarse en brazos del irracionalismo, sino por llevarla más allá de sí misma en una Ilustración de la Ilustración (Pecourt, 2007: 30).

\section{El campo social académico y la disposición "escolástica"}

Todos actuamos y pensamos dentro de una sociedad que el francés divide en "campos", que implican un habitus específico. Como señala el profesor L. E. Alonso, "El campo es el espacio social que se construye en torno a algo que es valorado socialmente. Este espacio es relacional y relativo, depende de las posiciones que lo circunscriben, y estas posiciones, a su vez, se apoyan en las diferentes dotaciones de capital con que cuentan los actores" (Alonso, 2002: 19). Por otra parte, el habitus en el espacio social es definido como "ese principio generador y unificador que retraduce las características intrínsecas y relacionales de una posición en un estilo de vida unitario, es decir un conjunto unitario de elección de personas, de bienes y de prácticas" (Bourdieu, 2007: 19). Los habitus son diferenciadores, en el sentido de que definen una posición y, además, crean las diferencias en la realidad social. En relación con los campos, puede haber habitus específicos que son como esquemas clasificatorios que subyacen al sentido práctico requerido por un campo y que se asume 
inconscientemente por el mero hecho de estar jugando el juego característico del campo en cuestión.

Un campo es, así pues, como una partida de cartas en la que se asumen unas reglas, se respetan o infringen también según ciertas pautas establecidas y se compite para obtener mayor ganancia, que es el "capital" en juego, no necesariamente económico. De hecho, igual que poseemos dinero, existen otros capitales, como el cultural, tan determinantes como el capital económico (Liozos, 2006), por lo que Bourdieu amplía el planteamiento marxista ortodoxo". Hay que tener en cuenta, entonces, que "La distribución de los agentes en el espacio social compromete, para ser exactos, tres dimensiones: el volumen global de capital poseído, la composición de este capital (peso relativo de los diferentes tipos de capital) y la trayectoria o evolución en el tiempo del volumen y composición del capital" (Inda y Duek, 2005: 4).

La noción bourdieusiana de habitus se refiere a ese sentido práctico que interviene antes de que, como nos hacen creer las proyecciones de la scholé y de su método científico, lo haga una razón consciente estratégica y calculadora (Peña y Rodríguez, 2002: 237-240; Follari, 2002: 120). Como campo específico con su correspondiente habitus, Bourdieu se refiere a la academia (scholé). Ésta reclama una actitud (habitus) de distanciamiento y frialdad respecto al mundo, una inercia, podríamos decir, objetivante. La sociología nos enseña que la mirada objetiva no es sino el producto de lo académico como campo social. Hay, por tanto, una "materia social" que posibilita el ejercicio y el nacimiento de esta mirada, que en este sentido aparece asociada a un tiempo y a unas instituciones sociales (Platón, la Academia). Se constituye, entonces, un universo dentro de la sociedad actual en el que lo escolar continúa vigente, como mentalidad, sentido y mirada (Bourdieu, 1999b: 29).

La mirada escolástica justifica, además, una posición social aristocrática, que parece elevarse por encima de sus propias condiciones y que niega su social razón de ser. Así, los privilegios y desigualdades quedan asegurados, en cuanto se pierden de vista en la perspectiva académica escolástica (Bourdieu, 1999b: 41). "Este aristocratismo [escolástico] debe su éxito al hecho de ofrecer a los habitantes de los universos escolásticos una 'teodicea de su privilegio' perfecta, una justificación absoluta de esa forma de olvido de la historia, el olvido de las condiciones sociales de posibilidad de la razón escolástica, que, a pesar de lo que en apariencia los separa, el humanismo universalista de la tradición kantiana comparte con los profetas desencantados del 'olvido del ser'" (Bourdieu, 1999b: 41). Así, la interpretación que Bourdieu realiza de Heidegger va en esta línea por la que el alemán cede a un aristocratismo intelectual que olvida su arraigo en unas condiciones sociales concretas, justo en la época de apogeo de las ciencias sociales que el alemán considera parte de la historia de caída y olvido del ser en el Occidente de la ciencia, la metafísica y la técnica (Bourdieu, 1991; 1999b: 43). Según éste, el tiempo y la historicidad del Dasein no pueden ser captados "científicamente" o "históricamente" (por parte de las ciencias

\footnotetext{
${ }^{2}$ Sobre las relaciones de Bourdieu con Marx, al que el francés acude en unos aspectos al tiempo que en otros lo rechaza, cf. Gutiérrez, 2003.
} 
históricas). En esto, "Heidegger presenta, en especial en sus obras de juventud, una manifestación particularmente aguda de hybris del pensamiento sin límites" (Bourdieu, 1999b: 44).

Lo que Bourdieu propone, por tanto, tanto a la filosofía como a la intelectualidad en general y al mundo de la scholé, es hacer consciente su origen y condiciones sociales como clase (Bourdieu, 1999b: 45; Moss, 2005) ${ }^{3}$, así como sus dinámicas de poder y lucha en las que coexisten una libido dominandi junto a la libido sciendi. De hecho, Bourdieu ha mostrado que concretamente en el campo de la universidad se da una lucha constante destinada a cambiar su estructura, en la que tanto el poder académico como el prestigio intelectual sirven de armas y objetos en pugna, al mismo tiempo (Bourdieu, 2008). Este tipo de análisis, lejos de verse como amenaza, por parte del filósofo o del universitario, ha de verse como un favor consistente en la puesta en evidencia de que la supuesta libertad y autonomía del pensamiento filosófico es resultado de un prejuicio (un habitus del campo filosófico). Algo que sin duda no gustará a los más "espiritualistas" y que será criticado por ellos desde "los valores sagrados de la persona y los derechos imprescriptibles del 'sujeto"" (Bourdieu, 1999b: 46). En este tipo de pensamiento, esclavo a juicio de Bourdieu de su propio campo de juego y habitus, se apela a lo intemporal y al fundamento (Bourdieu, 1999b: 46-47). Para combatir este idealismo, es preciso hacer ver que lo social es elemento constituyente del pensamiento aun en sus formulaciones más distanciadas y formalmente ahistóricas. En esto, y en otros momentos, Bourdieu parece apuntar con simpatía a los análisis de Foucault, al que, sin embargo, también cuestiona por su pathos causal-explicativo. Porque Bourdieu señala que la causalidad lineal de las explicaciones propia de la modernidad y su tendencia a los pares de opuestos es modernidad dentro de la postmodernidad. Este pathos moderno es originado históricamente y hay que detectarlo porque en ocasiones ciega para comprender y analizar cosas complejas y poliédricas como es la propia sociedad o espacio social, entendido como "(...) un sistema de posiciones sociales donde se definen unas en relación a otras" (Castón, 1996: 86).

Lo propio de la mirada científica (sociología, antropología) es el situarse en una relación de exterioridad ante el juego (campo social) que observa. Y la experiencia de la propia exteriorización le lleva a olvidar su propia vinculación a una práctica (Bourdieu, 1999b: 77-78). Pero si se aplica al propio científico o académico la mirada ejercitada en ver las prácticas ajenas puede abordarse el campo escolástico en su carácter de ritual y de espacio que proyecta distorsiones en la realidad que desde él es observada y estudiada. En definitiva, se sirve a la ciencia.

\footnotetext{
${ }^{3}$ Sin embargo, no podemos dejar de anotar que esta clave interpretativa ha sido considerada excesiva por algunos a la hora de abordar un pensamiento como el de Heidegger, por mucho que éste se asocie con ciertas ideas políticas perniciosas o con la situación social del intelectual elitista. Por ejemplo, Philipse (2002), a partir de la interpretación de Heidegger por parte de Bourdieu, sugiere que se utilicen además otros métodos de interpretación de las obras filosóficas, sin restringirse solamente a una sociología de la filosofía.
} 


\section{Razón y escuela. Matizando la Modernidad}

Como acabamos de indicar, Bourdieu pregunta certeramente cómo ignorar que el mundo escolástico está plagado de intereses sociales, por ejemplo de dominio, que se proyectan en la propia actividad científica (Bourdieu, 1999b: 91). Con dureza, Bourdieu declara: “(...) en la medida en que resulta posible gracias al privilegio, un privilegio que se ignora, la razón contiene en sí la virtualidad de un abuso de poder (...) es a la vez fuente de beneficios materiales y simbólicos e instrumento de dominación y legitimación" (Bourdieu, 1999b: 106). Su ventaja es prestar un velo de neutralidad y universalidad a lo que no es sino la más absoluta particularidad, es decir, hacer que lo arbitrario se presente como universal. Esto es achacado por Bourdieu a la filosofía tanto de Rawls como de Habermas (Bourdieu, 1999b: 107), quien "somete las relaciones sociales a una doble reducción o, lo que viene a ser lo mismo, a una doble despolitización, que lleva, sin que lo parezca, a replegar la política al terreno de la ética: Habermas reduce las relaciones de fuerza políticas a relaciones de comunicación (...)" (Bourdieu, 1999b: 91) .

La manera de liberar a la razón del olvido de su propio origen es el recurso bourdieusiano a la sociología de la scholé y de los intelectuales, así como a su conocida crítica a la escuela (Bourdieu y Passeron, 2001). En esto Bourdieu, aunque lo parezca, no debería ser acusado de relativista, ya que a lo que aspira es a una purga de los velos que cubren a la razón para que ésta muestre, verdaderamente, su efectividad, como hemos afirmado supra. Tampoco debe ser acusado de entender la escuela con una perspectiva fatalista que excluiría cualquier componente de resistencia, como se ha hecho a menudo confrontándolo con las teorías de la resistencia en pedagogía (Ávila, 2002) o con Foucault, de cuya teoría algún autor espera más posibilidades para la subversión que de la teoría bourdieusiana (Cronin, 1996) ${ }^{5}$. Bien es cierto que $L a$ reproducción parece no dejar muchas oportunidades a los agentes sociales dentro de la escuela para la transformación (Marqués, 2006: 88), pero en realidad, dentro de la

\footnotetext{
${ }^{4}$ Esta estrechez del planteamiento habermasiano frente al de Bourdieu es señalada también en algunas fuentes secundarias, como el trabajo de Schatzki (1987). No obstante, también se ha señalado alguna similitud entre Habermas y Bourdieu, en lo que se refiere a la concepción de la acción como aplicación de esquematismos a su vez emanados de la experiencia social y de la práctica. Cf. Maesschalck (2001). Según ambos, del contexto de la acción emanarían los significados que a su vez determinan a la acción.

${ }^{5}$ Hemos de añadir que nuestra postura personal discrepa de esta contraposición entre Foucault y Bourdieu en la que el segundo es acusado de no proveer de adecuados instrumentos para la transformación social. Por el contrario, como también opina Olson (1995), creemos que Bourdieu sí nos dota mejor de un arsenal emancipatorio. Bourdieu resulta excelente para llegar adonde Foucault sólo llega contradiciéndose a sí mismo, como señala la conocida crítica de Habermas a Foucault (Habermas, 2008: 291-319), ya que todo discurso emancipatorio requiere aquello que el propio Foucault elimina tajantemente (un punto de apoyo arquimédico y principios a partir de los cuales ejercer la crítica). Bourdieu, en sus matizados elogios a la razón y a la ciencia sí se sitúa con claridad, pese a todo, en la tradición crítica, emancipatoria y universalista de la Modernidad.
} 
propia academia y, quizás, incluso dentro de la escuela cabría pensar en formas de superar la inercia reproduccionista (Mills, 2008; Nash, 2004) ${ }^{6}$

Lo que Bourdieu pone de manifiesto es cómo la escuela sirve a la sociodicea (la justificación de la estructura social vigente): "El mito del 'don natural' y el racismo de la inteligencia ocupan el centro de una sociodicea, vivida íntimamente por todos los dominantes, más allá de las diferencias en los compromisos éticos o políticos proclamados, que erige la 'inteligencia' (medida escolarmente) en principio supremo de legitimación y ya no imputa la pobreza y el fracaso -en una civilización de los 'resultados', en la que hay que tener éxito en todo- a la pereza, la imprevisión y el vicio, sino a la estupidez" (Bourdieu, 1999b: 108). Por eso, señala A. B. Gutiérrez: "Así, en el orden de las prácticas culturales, muestra Bourdieu, la cultura dominante, haciéndose reconocer como universal, legitima los intereses del grupo dominante, forzando a las otras culturas a definirse negativamente por relación a ella" (Gutiérrez, 2004: 297).

Así pues, el elemento más interesante de Bourdieu para ser aplicado a la teoría de la educación es la concepción histórica de la razón, es decir, su concepción de que los fundamentos de la razón son históricos y proceden de la estructuración altamente compleja, multidimensional, de la sociedad. Esto hace que relativice constantemente las ideas o valores en apariencia más neutros y objetivos, señalando lo arbitrario de las mismas, contra la fe en su origen argumentativo, racional o lógico. Pero, contra toda permanencia en un escepticismo inmovilizante, indica que "paradójicamente, tal vez someter a la razón a la prueba de la historicización más radical, en particular echando por tierra la ilusión del fundamento al recordar lo arbitrario del origen y mediante la crítica histórica y sociológica de los instrumentos de la propia ciencia histórica y sociológica, sea la manera de liberarla de la arbitrariedad y la relativización histórica" (Bourdieu, 1999b: 125).

Bourdieu advierte que la legitimación de figuras, personas, políticos, obra mediante el olvido de la arbitrariedad que funda el poder o la presencia de esa figura. Así, un intelectual o un político estarán más legitimados según este engaño esté mejor realizado. El poder para Bourdieu, contra la visión más piramidal y monolítica del marxismo, está diluido, de un modo semejante a como lo entiende Foucault, frente a la concepción marxista: "sólo se realiza y se manifiesta a través de un conjunto de campos unidos por una verdadera solidaridad orgánica y, por lo tanto, diferentes e interdependientes a la vez. Más precisamente, se ejerce, de forma invisible y anónima, mediante acciones y reacciones, anárquicas en apariencia, pero, de hecho, impuestas estructuralmente, de agentes e instituciones incluidos en campos a la vez competidores y complementarios, como, por ejemplo, el económico y el escolar, e implicados en el circuito de intercambios legitimadores cada vez más dilatados y complejos y, por lo tanto, cada vez más eficaces simbólicamente, pero que asimismo dejan, en medida

\footnotetext{
${ }^{6}$ Ciertamente, también puede afirmarse que Bourdieu parece haber defendido ambas cosas (por un lado, el férreo determinismo de la teoría reproduccionista, y, por otro lado, la posibilidad de que mediante acciones políticas o pedagógicas puedan cambiarse las cosas), a lo largo de su evolución intelectual, como señala en su excelente análisis Van Zanten (2005).
} 
creciente, cada vez más espacio, al menos en potencia, a los conflictos de poder y autoridad" (Bourdieu, 1999b: 137). Así, el poder sería un fenómeno multidimensional, variable, fluctuante, asociado al funcionamiento de un campo social, como ha llegado a mostrarse incluso en la emergencia social de la figura del dictador (Van der Linde, 2007).

Puede haber, en el contexto de lucha entre dominadores y dominados, teorías, como la de la lucha de clases o la división de los tres poderes, que se desarrollen con el efecto de influir en estas luchas y de reconfigurar el panorama del dominio social. Pero siempre será común que el dominador debe su legitimación al olvido de la arbitrariedad que lo instituye, ocultando la verdadera motivación e intereses reales, variables y de muy distinta índole, que lo mueven para dominar. En este sentido, el derecho consagra, y ésa es su función principal según Bourdieu, la configuración del dominio en una sociedad. Surgen instituciones específicas para legitimar y asegurar el dominio, como es la escuela, pero que, como en este caso, cuanto más complejas y autónomas son, más lugar reservan también para la aparición de elementos de subversión (Bourdieu, 1999b: 140-141).

Así pues, hay lugar en el entramado social, siguiendo la teoría de Bourdieu, también para subversiones y cambios. Bourdieu pretende con esto conciliar el absolutismo logicista con el relativismo historicista. La razón puede emancipar sin perder por ello su carácter histórico y contingente (contra Habermas). Pero, también, se puede defender que la historia desde su juego de luchas e intereses ha podido producir algo (la razón, el pensamiento argumentativo) que sin dejar de ser histórico ostenta un carácter objetivo, "universal", distanciado que le faculta para impugnaciones y contribuciones a la transformación social (contra Foucault). Así, la observación (sociológica) de lo que ocurre de hecho en el mundo científico, “(...) obliga a adherirse a un realismo crítico y reflexivo, en ruptura a la vez con el absolutismo epistémico y el relativismo irracionalista" (Bourdieu, 1999b: 147).

La pretensión de tomar conciencia de la historicidad de la razón, según Bourdieu, "Significa negarse a reemplazar el Dios creador de las 'verdades y los valores eternos' por el Sujeto creador y devolver a la historia, y a la sociedad, lo que se ha atribuido a una trascendencia o un sujeto trascendental" (Bourdieu, 1999b: 152). Así pues, lo que Bourdieu no se cansa de combatir es un fetichismo platonizante de la razón propio del pensamiento escolástico (de la scholé). Por eso, hay además un anti-kantismo en Bourdieu, parejo a una fuerte crítica del idealismo constructivista, del sujeto que conoce y se hace de modo absoluto, lo que interesa sobremanera a la pedagogía para valorar a fondo y tal vez matizar, por ejemplo, algunos discursos que se dice se basan en una concepción pedagógica constructivista ${ }^{7}$. Por el contrario, y como es

\footnotetext{
${ }^{7}$ En particular, la teoría de las competencias, que se dice está basada en teorías constructivistas, desde la teoría sociológica de Bourdieu habría de replantearse. En efecto, parece que hay instrumentos para obligarnos a cuestionar gran parte del discurso de las competencias en pedagogía, como certeramente señalan Campillo et al. (2008). En general, la teoría de Bourdieu desmiente numerosos dogmas y pseudo verdades del pensamiento neoliberal, en lo que éste tiene de ensalzamiento de la razón estratégica y calculadora (técnico instrumental) propia de un 
frecuentemente olvidado por este discurso, "Hay que buscar en el objeto elaborado por la ciencia (el espacio social o el campo) las condiciones sociales de posibilidad del 'sujeto' y su actividad de elaboración del objeto (...) y revelar de este modo los límites sociales de sus actos de objetivación” (Bourdieu, 1999b: 159).

\section{EI sujeto y la illusio}

Los agentes sociales ocupan siempre una posición en el espacio social definida por la acumulación de distintas especies de capital, la posición relativa y la distancia respecto a otros agentes sociales (llamada por Bourdieu "distinción"), el tiempo en cuanto generador de rentas materiales o simbólicas, localizaciones físicas (barrios, etc.) y extensión de las mismas (propiedades materiales, p. e.). Se produce además una illusio en el agente que percibe como normal lo arbitrario de su posición, debido al ajuste entre las condiciones sociales y su habitus. O sea, sus estructuras cognitivas son conformadas por la estructura del espacio social. Esto implica que para Bourdieu no hay un determinismo que anule la contribución del sujeto a la construcción del mundo, pero tampoco el subjetivismo idealista por el que el sujeto dispondría de una voluntad omnipotente (Longa, 2009: 210-213). El habitus implica en realidad ambas cosas: una constitución del agente social por sus condiciones sociales de existencia y, al mismo tiempo, un poder clasificatorio, unificador y elaborador de la realidad desde su cuerpo socializado (Bourdieu, 1999b: 181).

No obstante, la lectura escolástica que sigue a la tradición platónica tiende a considerar lo corporal, en lo que se inscriben los habitus, como un obstáculo para la comprensión. El sujeto es absolutamente creativo y seguiría una intención a partir de finalidades establecidas racionalmente. La visión escolástica que tiende al distanciamiento, se proyectó en las distintas concepciones del hombre o el sujeto y generó dos corrientes en apariencia contradictorias: el mecanicismo determinista y el finalismo de la libertad incondicionada. Contra ciertas teorías de la economía o la

sujeto que desde su voluntad y consciencia se adaptaría a una realidad que se presenta como absoluta frente a la arbitrariedad que en el fondo la constituye. Así, hay una visión de tono idealista y racionalista que oculta las irracionalidades y elementos inerciales e inconscientes de la toma humana de decisiones y del uso de la razón estratégica. En esto también ha puesto el punto de mira el marxismo, como certeramente señala en su excelente exposición de El Capital los profesores Carlos Fernández Liria y Luis Alegre Zahonero (2010). Estos autores señalan que la idea de racionalidad como cálculo egoísta propia del liberalismo capitalista es ya ideológica, o sea, producto del olvido de las condiciones sociales que opera justificando dichas condiciones. Además, lejos de promover el fatalismo resignado, los estudios de Bourdieu ofrecen muy válidos elementos científicos para emprender una lucha consciente para la transformación social (Bourdieu, 1999a; Barranco, 2003: 206-210; Álvarez, 2005). Sobre Bourdieu como crítico del neoliberalismo junto a otros autores, cf. Vergara (2005). En Bourdieu encontramos, desde luego, elementos para pensar las condiciones sociales requeridas para la democracia y la justicia social, lo que lo convierten en un pensador político (Wacquant, 2004) muy crítico con ciertos idealismos encubridores como el de los valores de libertad, igualdad y justicia social esgrimidos constantemente en nuestros sistemas políticos (Topper, 2001). 
filosofía política liberal, el hombre no decide estratégica y racionalmente su acción, sino que, según Bourdieu, actúa por una inercia en gran parte inconsciente en la que interviene la disposición del sujeto y el mundo social que habita y lo determina (Bourdieu, 1999b: 183). Así, “(...) el agente no es nunca del todo el sujeto de sus prácticas: mediante las disposiciones y la creencia de que originan la implicación en el juego, todos los presupuestos constitutivos de la axiomática del campo (la dóxa epistémica, por ejemplo) se introducen incluso en las intenciones en apariencia más lúcidas" (Bourdieu, 1999b: 184). Así, el sentido práctico permite obrar "como es debido" sin la necesidad de un deber ser de tipo kantiano o regla de comportamiento (Bourdieu, 2008a). El agente actúa en un ajuste inconsciente con su campo, anticipando corporalmente las tendencias del campo y los comportamientos producto del habitus específico del mismo.

Así, tampoco corresponde a la realidad descrita por el sociólogo francés la concepción marxista de ideología en lo que ésta pueda tener de universo de creencias e ideas que habitan la conciencia de un sujeto. No es necesario, por tanto, emplear la noción marxista de "falsa conciencia" para explicar el ajuste de los agentes sociales con su mundo ${ }^{8}$. El orden social se inscribe en los cuerpos a través de esta confrontación permanente, más o menos dramática, pero que siempre otorga un lugar destacado a la afectividad y, más precisamente, a las transacciones afectivas con el entorno social (Bourdieu, 1999b: 186). Esto es genialmente expresado, recuerda Bourdieu, por Kafka en su cuento "En la colonia penitenciaria", en la que la norma social se graba mediante una máquina que araña la piel en el cuerpo de los hombres (Bourdieu, 1999b: 187). Así el agente social se siente en casa porque el mundo está dentro de él en la forma de habitus que produce prácticas y la sensación de un fatum (destino). Señala más adelante: "El cuerpo está en el mundo social, pero el mundo social está en el cuerpo (en forma de héxis y de eîdos). Las propias estructuras del mundo están presentes en las estructuras (o, mejor aún, en los esquemas cognitivos) que los agentes utilizan para comprenderlo" (Bourdieu, 1999b: 199-200).

Pero también ocurren desajustes, en lugares límite del espacio social, que permiten la posición distanciada respecto a las creencias específicas de un campo y sus jerarquías, así como disponen a la herejía. "Se observa así que a posiciones contradictorias, aptas para ejercer sobre sus ocupantes 'dobles coerciones' estructurales, corresponden a menudo habitus desgarrados, dados a la contradicción y la división contra sí mismos, generadora de sufrimiento" (Bourdieu, 1999b: 210). Esto ocurre cuando las disposiciones (habitus) no casan con el campo, lo que pone en evidencia la normatividad arbitraria que rige cada campo y que cuando no hay fricciones permanece invisible, como algo dado o normal. Una típica situación de este tipo es, por ejemplo, la del "nuevo rico".

A la hora de explicar cómo se genera la illusio, ese ajuste perfecto entre disposiciones (habitus) y condiciones sociales, Bourdieu acude a la psicología. Habría

\footnotetext{
${ }^{8}$ Por ejemplo, se ha explicado a partir de Bourdieu el fenómeno que se da en las relaciones
} escolares y educativas que suele denominarse "doble discurso" (Mazzola, 2000). 
que ir al proceso de crecimiento de cada individuo para ver en su historia familiar y personal las causas de su ajuste casi perfecto con el medio. Creo que de manera acertada, el sociólogo francés apunta a la necesidad de reconocimiento y aprobación como la fuerza que nos conduce a satisfacer los requerimientos del medio. Se trata de un proceso largo, constante, imperceptible, sancionado por ritos de institución (como los exámenes) que se inicia en la infancia e incluso antes del nacimiento. El resultado es que el agente social escogerá a la institución que, en realidad, lo ha escogido a él. La psicología (psicoanálisis) debe explicar por qué motivaciones profundas se "escoge" invertir en un campo o institución, por qué se juega un juego. Pero sobre todo, es por la búsqueda de reconocimiento que uno se ajusta al medio en el que desea ser incluido. Así se explica la fuerza del denominado por Bourdieu "capital simbólico" (Bourdieu, 1999b, p. 220).

\section{Conclusión: potencial emancipatorio de la ciencia}

Según el francés, gran parte de la educación consiste en hacer creer el valor de determinado capital simbólico por el que el agente social regirá su existencia (social). Es mediante una labor educativa como se provoca la transformación de los cuerpos en los que se inscribe la disposición (habitus) hacia la estructura social. El cuerpo, pues, participa cómplicemente del juego social. "El reconocimiento práctico a través del cual los dominados contribuyen, a menudo sin saberlo y, a veces, contra su voluntad, a su propia dominación al aceptar tácitamente, por anticipado, los límites impuestos, adquiere a menudo la forma de la emoción corporal (vergüenza, timidez, ansiedad, culpabilidad), con frecuencia asociada a la impresión de regresar hacia relaciones arcaicas, las de la infancia y el universo familiar" (Bourdieu, 1999b: 223-224). El cuerpo, pues, se hace cómplice de la dominación y expresa la violencia de muchas estructuras sociales, viviéndose la fractura social como contradicción entre la voluntad y el cuerpo (Bourdieu, 1999b: 224). Así, Bourdieu define la violencia simbólica como "(...) esa coerción que se instituye por mediación de una adhesión que el dominado no puede evitar otorgar al dominante ( $\mathrm{y}$, por lo tanto, a la dominación) cuando sólo dispone, para pensarlo y pensarse o, mejor aún, para pensar su relación con él, de instrumentos de conocimiento que comparte con él y que, al no ser más que la forma incorporada de la estructura de la relación de dominación, hacen que ésta se presente como natural;" (Bourdieu, 1999b: 224-225). Es decir, el dominado incorpora los esquemas clasificatorios para percibirse y evaluarse y para percibir y evaluar a los dominantes, que proceden de la sociedad donde se da la dominación. Así que "esa sumisión nada tiene que ver con una relación de 'servidumbre voluntaria' y esa complicidad no se concede mediante un acto consciente y deliberado" (Bourdieu, 1999b: 225). Por esto, no funciona como acción liberadora, según Bourdieu, la mera toma de conciencia que no cuenta, necesariamente, con la complicidad del cuerpo dominado, frente a la tradición marxista de la ideología y la concientización. La dominación se da como una extraordinariamente fuerte inercia que no se suprime a golpe de pensamiento y de conciencia.

Así pues, como resultado de una violencia simbólica que se realiza sobre todo a través de la escuela (Ávila, 2005: 163-164; Martínez García, 2007: 294; Nash, 2005), 
se crea la illusio que percibe el orden instituido como "natural", como de sentido común, como algo que cae por su propio peso. "En La reproducción, frente a una supuesta teoría que defiende la existencia de igualdad ante la enseñanza y que no existen, por tanto, determinaciones sociales, sino sencillamente distintos resultados en función de las 'voluntades' y 'capacidades' individuales, los señalados autores analizan cómo incluso las voluntades y las capacidades están determinadas por la clase social" (Álvarez Sousa, 1996: 161). Esto se da con fuerza incluso en quienes han sufrido los peores efectos del régimen de dominación. El situarse al margen del lugar de los privilegios no garantiza necesariamente la subversión o la protesta (Bourdieu, 1999b: 228). En este proceso resulta fundamental, y Bourdieu lo repite bastante, el papel del Estado y de quienes lo controlan.

El Estado es la institución por excelencia que sirve a la dominación debido a la capacidad extraordinaria que tiene de imponer silenciosa y subrepticiamente un punto de vista, sin necesidad del constante recurso a la fuerza. Toda experiencia primera del mundo, en este sentido y contra los fenomenólogos, "constituye una relación socialmente elaborada, como los esquemas perceptivos que la posibilitan" (Bourdieu, 1999b: 230). El Estado, apoyado en la familia y en la Escuela, “(...) crea las condiciones de una sintonización inmediata de los habitus que constituye a su vez el fundamento de un consenso sobre este conjunto de evidencias compartidas que son constitutivas del sentido común" (Bourdieu, 1999b: 231). El Estado impone con fuerza las estructuras cognitivas por las cuales es percibido, valiéndose de, entre otras cosas, la coherencia del universo simbólico que transmite, como se ve en el campo jurídico en el que se apoya. Así, el reconocimiento de su legitimidad se basa en el ajuste entre la estructura social y la estructura (social) incorporada en forma de habitus. Para entender bien esto, desde luego, hay que abandonar aquello a lo que Bourdieu se refiere como filosofía de la conciencia (el marxismo, entre otras). "El ajuste prerreflexivo entre las estructuras objetivas y las incorporadas, y no la eficacia de la propaganda deliberada de los aparatos, o el libre reconocimiento de la legitimidad por los ciudadanos, explica la facilidad, en definitiva realmente asombrosa, con la que, a lo largo de la historia, y exceptuando contadas situaciones de crisis, los dominantes imponen su dominación" (Bourdieu, 1999b: 234).

Por eso, la lucha contra la opresión no puede plantearse desde los discursos, la "conversión de los espíritus" mediante la educación, la predicación racional. Estos son prejuicios y actitudes características del pathos intelectual que hemos descrito en este trabajo y que obedecen a las condiciones específicas de su campo. "El habitus, indudablemente, no es un destino, pero la acción simbólica no puede, por sí sola, y al margen de cualquier transformación de las condiciones de producción y fortalecimiento de las disposiciones, extirpar las creencias corporales, pasiones y pulsiones que permanecen por completo indiferentes a las conminaciones o las condenas del universalismo humanista (que, a su vez, por lo demás, también arraigan en disposiciones y creencias)" (Bourdieu, 1999b: 237). Así que hay que conceder este papel liberador a la ciencia, según Bourdieu: "Sólo la crítica histórica, arma capital de la introspección, puede liberar el pensamiento de las imposiciones que se ejercen sobre él cuando, dejándose llevar por las rutinas del autómata, trata como si fueran cosas unas construcciones históricas cosificadas" (Bourdieu, 1999b: 240). 


\section{Referencias bibliográficas}

ALONSO, L. E. (2002). Pierre Bourdieu In memoriam (1930-2002). Entre la bourdieumanía y la reconstrucción de la sociología europea, Revista española de investigaciones sociológicas, 97, 9-28.

ÁlVAREZ, A. N. (2005). La globalización según Bourdieu, Cuadernos de Trabajo Social, 18, 121-135.

ÁLVAREZ SOUSA, A. (1996). El constructivismo estructuralista: la teoría de las clases sociales de Pierre Bourdieu, Revista española de investigaciones sociológicas, 75, 145-172.

ÁVILA, M. (2002). Violencia estructural y resistencia: dos caras de la misma moneda, Revista Electrónica Interuniversitaria de Formación del Profesorado, 5 (2). Consultado (27/11/2010) en

http://web.archive.org/web/20041217225306/www.aufop.org/publica/reifp/articulo. asp?pid $=209 \&$ docid $=888$

ÁVILA, M. (2005). Socialización, Educación y Reproducción Cultural: Bourdieu y Bernstein, Revista Interuniversitaria de Formación del Profesorado, 19 (1), 159174.

BARRANCO, O. (2003). Aportacions de Pierre Bourdieu a la 'teoria de la ideologia', Revista Catalana de Sociologia, 19, 189-217.

BOURDIEU, P. (1991). La ontología política de Martin Heidegger. Barcelona: Paidós.

BOURDIEU, P. (1999a). Contrafuegos. Reflexiones para servir a la resistencia contra la invasión neoliberal. Barcelona: Anagrama.

BOURDIEU, P. (1999b). Meditaciones pascalianas. Barcelona: Anagrama.

BOURDIEU, P. (2007). Razones prácticas. Sobre la teoría de la acción. Barcelona: Anagrama.

BOURDIEU, P. (2008). Homo Academicus. Madrid: Siglo XXI.

BOURDIEU, P. y PASSERON, J. Cl. (2001). La reproducción. Elementos para una Teoría del Sistema de Enseñanza. Madrid: Editorial Popular.

CAMPILLO, M. J. et al. (2008). Actores y campos: de lo científico y lo profesional, Red U. Revista de Docencia Universitaria, numero monográfico I1 "Formación centrada en competencias (II)". Consultado (27, 11, 2010) en http://www.redu.m.es/Red_U/m2

CASTÓN, P. (1996). La sociología de Pierre Bourdieu, Reis. Revista española de investigaciones sociológicas, 76, 75-98.

CRONIN, C. (1996). Bourdieu and Foucault on Power and Modernity, Philosophy and Social Criticism, 22 (6), 55-85. 
GARCÍA DEL DUJO, A. y MÍNGUEZ, R. (2011). Los límites de la educación en valores cívicos: cuestiones y propuestas pedagógicas, Educación XX1, 14 (2), 263285.

FERNÁNDEZ LIRIA, C. Y ALEGRE ZAHONERO, L. (2010). El orden de El Capital. Por qué seguir leyendo a Marx. Madrid: Akal.

FERRANTE, C. (2008a). Corporalidad y temporalidad: Fundamentos fenomenológicos de la teoría Práctica de Pierre Bourdieu, Nómadas. Revista Crítica de Ciencias Sociales y Jurídicas, 20. Consultado (28/11/2010) en

http://www.ucm.es/info/nomadas/20/carolinaferrante.pdf

FERRANTE, C. (2008b). De Mauss a Levi-Strauss: la concepción de lo social como doble verdad en Pierre Bourdieu, Intersticios. Revista Sociológica de Pensamiento Crítico, 2 (2), 49-61. Consultado (27/11/2010) en http://www.intersticios.es/article/view/2736/2123.

FOLLARI, R. (2002). Pierre Bourdieu y la complejidad de lo social, Utopía y praxis latinoamericana, 7 (16), 119-121.

GUTIÉRREZ, A. B. (2003). Con Marx y contra Marx: el materialismo en Pierre Bourdieu, Revista Complutense de Educación, 14 (2), 453-482

GUTIÉRREZ, A. B. (2004). Poder, habitus y representaciones: recorrido por el concepto de violencia simbólica en Pierre Bourdieu, Revista Complutense de Educación, 15 (1), 289-300.

HABERMAS, J. (2008). El discurso filosófico de la modernidad. Buenos Aires: Katz Editores.

INDA, G. y DUEK, C. (2005). El concepto de clases en Bourdieu: ¿nuevas palabras para viejas ideas?, Aposta. Revista de ciencias sociales, 23. Consultado (28/11/2010) en http://www.apostadigital.com/revistav3/hemeroteca/indayduek.pdf

LIOZOS, S. (2006). Capital cultural y social: ¿qué podemos aprender para investigar y reforzar la colaboración entre familia y escuela?, C\&E: Cultura y Educación, 18 (34), 219-230.

LÓPEZ HERRERÍAS, J. A. (2010). ¿Qué 'Yo' es valioso para el mundo de hoy?, Teoría de la Educación. Revista Interuniversitaria, 223 (1), 65-90.

MACEDO, D. (2000). Una pedagogía antimétodo. Una perspectiva freiriana, Educación XX1, 3, 53-61.

MAESSCHALCK, M. (2001). La reduction du contexte chez Bourdieu et Habermas, Revue Philosophique de Louvain, 99 (2), 254-290.

MARCOULATOS, I. (2001). Merleau-Ponty and Bourdieu on Embodied Significance. Journal for the Theory of Social Behaviour, 31 (1), 1-27.

MARQUÉS, I. (2006). Bourdieu o el 'caballo de Troya' del estructuralismo, Reis. Revista española de investigaciones sociológicas, 115, 69-100. 
MARTÍNEZ GARCÍA, J. S. (2007). Clase social, género y desigualdad de oportunidades educativas, Revista de Educación (Madrid), 342, 287-306.

MAZZOLA, C. (2000). El doble discurso como práctica institucional. Un análisis desde Pierre Bourdieu, Fundamentos en Humanidades, 1 (1), 80-89.

MEDINA, J. (2003). Wittgenstein and Nonsense: Psychologism, Kantianism, and the Habitus, International Journal of Philosophical Studies, 11 (3), 293-318.

MILLS, C. (2008). Reproduction and Transformation of Inequalities in Schooling: The Transformative Potential of the Theoretical Constructs of Bourdieu, British Journal of Sociology of Education, 29 (1), 79-89.

MORENO, J. L. y VÁZQUEZ, F. (2006). Presentación. 'Serían necesarios comandos de intervención filosófica rápida', 7-20, en J. L. Moreno y F. Vázquez (Eds). Pierre Bourdieu y la Filosofia. Barcelona: Montesinos.

MOSS, P. A. (2005). Toward Epistemic Reflexivity in Educational Research: A Response to Scientific Research in Education, Teachers College Record, 107 (1), 19-29.

NASH, R. (2004). Can the Arbitrary and The Necessary Be Reconcilied? Scientific Realism and the School Curriculum, Journal of Curriculum Studies, 36 (5), 605623.

NASH, R. (2005). Cognitive "Habitus" and Collective Intelligence: Concepts for the Explanation of Inequality of Educational Opportunity, Journal of Education Policy, $20(1), 3-21$.

OLSON, K. (1995). Habitus and Body Language: Towards a Critical Theory of Symbolic Power, Philosophy and Social Criticism, 21 (2), 23-49.

PECOURT, J. (2007). El intelectual y el campo cultural. Una variación sobre Bourdieu, Revista Internacional de Sociología, 65 (47), 23-43.

PEÑA, J. V. y RODRÍGUEZ, M. C. (2003). Identidades esquemáticas de género en la escuela: a propósito del primer aniversario de la muerte de Pierre Bourdieu, Teoría de la Educación. Revista Interuniversitaria, 14, 235-263.

PHILIPSE, H. (2002). Questions of Method: Heidegger et Bourdieu, Revue Internationale de Philosophie, 56 (220), 275-298.

PRIETO, M. (2011). La vida afectiva en la Teoría de la Educación. El tratamiento de las emociones en el Seminario Interuniversitario de Teoría de la Educación, Teoría de la Educación. Revista Interuniversitaria, 23 (1), 107-129.

SANTOS, M. (2008). Ideas filosóficas que fundamentan la pedagogía de Paulo Freire, Revista Iberoamericana de Educación, 46, 155-173.

SANTOS, M. (2010). Pedagogía, historia y alteridad, Teoría de la Educación. Revista interuniversitaria, 22 (2), 63-84. 
SANTOS, M. (2011a). Filosofía de la educación liberadora, 143-163, en Gervilla, E., Jiménez, F. J. y Santos, M. (eds). La educación nos hace libres. La lucha contra nuevas alienaciones. Madrid: Biblioteca Nueva.

SANTOS, M. (2011b). Limitaciones de la pedagogía de John Dewey, Bordón. Revista de Pedagogía, 63 (3), 121-130.

SCHATZKI, Th. R. (1987). Overdue Analysis of Bourdieu's Theory of Practice. Inquiry: An Interdisciplinary Journal of Philosophy, 30, 113-135.

TOPPER, K. (2001). Not So Trifling Nuances: Pierre Bourdieu, Symbolic Violence, and the Perversions of Democracy, Constellations: An International Journal of Critical and Democratic Theory, 8 (1), 30-56.

VAN Der LINDE, C. G. (2007). Mosaico intercultural de abusos de poder, Cuadernos de Filosofía Latinoamericana, 28 (96), 91-109.

VAN ZANTEN, A. (2005). Bourdieu as Education Policy Analyst and Expert: A Rich but Ambiguous Legacy, Journal of Education Policy, 20 (6), 671-686.

VERGARA, J. (2005). La utopía neoliberal y sus críticos, Utopía y Praxis Latinoamericana, 10 (31), 37-62.

WACQUANT, L. (2004). Pointers on Pierre Bourdieu and Democratics Politics, Constellations: An International Journal of Critical and Democratic Theory, 11 (1), 3-15.

\section{Correspondencia con el autor:}

Marcos SANTOS GÓMEZ

Departamento de Pedagogía

Facultad de Ciencias de la Educación

Universidad de Granada

Campus Universitario de Cartuja S/N

18071 Granada

Teléfono.: 958246195

email: masantos@ugr.es 